Received: 23 February 2018

Accepted: 3 May 2018

Published online: 30 May 2018

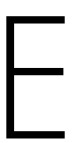

C
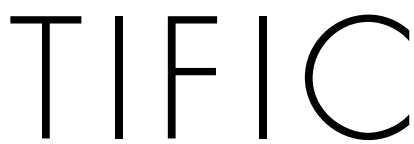

REPSO

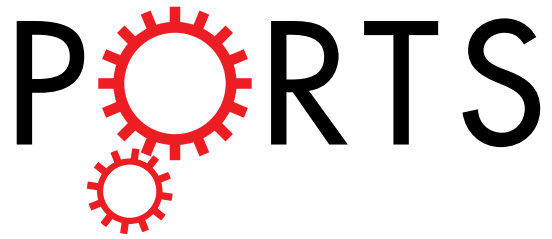

OPEN

\section{The A/T/N biomarker scheme and patterns of brain atrophy assessed in mild cognitive impairment}

\author{
Urban Ekman ${ }^{(1)}{ }^{1}$, Daniel Ferreira ${ }^{1}{ }^{1}$ \& Eric Westman $\mathbb{D}^{1,2}$
}

The objective of this study was to evaluate the $A / T / N$ biomarker scheme in relation with brain atrophy patterns in individuals with mild cognitive impairment $(\mathrm{MCl})$. Of the 154 participants with $\mathrm{MCl}, 74$ progressed to $A D$ within 36 -months, and 80 remained stable. In addition, 101 cognitively healthy participants and 102 participants with $A D$ were included. The $A / T / N$ classification was assessed with cerebrospinal fluid markers. Each individual was rated as either positive (abnormal) or negative (normal) on each biomarker. Brain atrophy was assessed with visual ratings from magnetic resonance imaging. None of the individuals with $\mathrm{MCl}$ progressed to $A D$ if they had a negative " $A$ " biomarker in conjunction with minimal atrophy. In contrary, several individuals with $\mathrm{MCl}$ progressed to AD if they had a positive " $\mathrm{A}$ " biomarker in conjunction with minimal atrophy. Numerous individuals with $\mathrm{MCl}$ showed inconsistency in the neurodegeneration domain ("N") regarding t-tau and atrophy. The assessment of the $A / T / N$ classification scheme in addition with brain atrophy patterns in $\mathrm{MCl}$, increases the knowledge of the clinical trajectories and the variability within the neurodegeneration domain. This emphasises that individuals with $\mathrm{MCl}$ display heterogeneous longitudinal patterns closely connected to their biomarker profiles, which could have important clinical implications.

The clinical manifestations of mild cognitive impairment (MCI) are associated with diverse trajectories ${ }^{1-3}$. The addition of biomarkers might enhance predictions of the longitudinal development from MCI to Alzheimer's disease $(\mathrm{AD})^{4,5}$. The $\mathrm{A} / \mathrm{T} / \mathrm{N}$ binary biomarker classification scheme has recently been proposed, aiming to be easily applicable on an individual level ${ }^{6}$. In keeping with the National Institute on Aging Alzheimer's Association $(\mathrm{NIA}-\mathrm{AA})^{7}$ and the International Working Group (IWG) ${ }^{8}$, the "A" class corresponds with an amyloid beta $(\mathrm{A} \beta)$ biomarker; the "T" class with a tau biomarker; and "N" with a neurodegeneration biomarker. Cerebrospinal fluid (CSF) biomarkers have the advantage that all A/T/N categories can be measured. Another way of assessing the "N" domain is to visually rate atrophy in the brain using rating scales for magnetic resonance imaging (MRI). Visual rating scales are commonly applied in specialized clinical settings ${ }^{9}$ and allow identification of different AD-related patterns of atrophy ${ }^{10-13}$. The patterns of atrophy resemble the distribution of neurofibrillary tangles $(\mathrm{NFT})^{14}$ in the brain. This may help to reach a greater understanding of the mechanisms underlying heterogeneity and disease progression in $\mathrm{AD}$. Translating atrophy patterns related to $\mathrm{AD}$-subtypes and the $\mathrm{A} / \mathrm{T} / \mathrm{N}$ classification scheme to patients with MCI is of utmost importance. We evaluated the $\mathrm{A} / \mathrm{T} / \mathrm{N}$ classification scheme using CSF markers in individuals with MCI that either progressed to AD or remained stable. In addition, we studied brain atrophy patterns generated from visual rating scales in combination with the $\mathrm{A} / \mathrm{T} / \mathrm{N}$ classification, with a special focus on variability in the "N" domain.

\section{Methods}

Study population. Participants were included from the Alzheimer's Disease Neuroimaging Initiative (ADNI), which was launched in 2003 by the National Institute on Aging, the National Institute of Biomedical Imaging and Bioengineering, the Food and Drug Administration, private pharmaceutical companies, and non-profit organisations ${ }^{11}$. Only data from the ADNI-1 cohort were included in the current study. The clinical diagnostic procedure has previously been described ${ }^{15}$. In summary, healthy controls (HC) had no memory complaints, and had a Clinical Dementia Rating $(\mathrm{CDR})^{16}$ score of 0 , normal performance on objective cognitive

${ }^{1}$ Division of Clinical Geriatrics, Department of Neurobiology, Care Sciences, and Society, Karolinska Institutet, Stockholm, Sweden. 2Department of Neuroimaging, Centre for Neuroimaging Sciences, Institute of Psychiatry, Psychology and Neuroscience: King's College London, London, UK. Correspondence and requests for materials should be addressed to U.E. (email: urban.ekman@ki.se) 


\begin{tabular}{|l|l|l|l|l|}
\hline & $\begin{array}{l}\text { HC } \\
(\mathbf{n}=101)\end{array}$ & $\begin{array}{l}\text { MCI-S } \\
(\mathbf{n}=\mathbf{8 0})\end{array}$ & $\begin{array}{l}\text { MCI-P } \\
(\mathbf{n = 7 4 )}\end{array}$ & $\begin{array}{l}\text { AD } \\
(\mathbf{n}=\mathbf{1 0 2})\end{array}$ \\
\hline Age & $75,46(5,18)$ & $74,49(7,15)$ & $74,51(7,29)$ & $74,96(7,91)$ \\
\hline Years of education & $15,61(2,90)$ & $16,15(2,89)$ & $15,66(3,13)$ & $15,13(3,29)$ \\
\hline MMSE* & $29,05(1,05)$ & $27,19(1,66)$ & $26,49(1,82)$ & $23,55(1,89)$ \\
\hline AVLT delayed* & $7,19(3,55)$ & $3,33(3,29)$ & $1,42(2,04)$ & $0,95(1,94)$ \\
\hline A $\beta_{42}{ }^{*}$ & $206,86(53,78)$ & $177,92(57,94)$ & $147,44(37,97)$ & $142,46(39,61)$ \\
\hline p-tau* & $24,83(13,96)$ & $30,58(15,72)$ & $40,35(16,70)$ & $41,76(19,77)$ \\
\hline t-tau* & $69,12(27,89)$ & $85,96(43,21)$ & $115,77(56,84)$ & $121,52(57,45)$ \\
\hline APOE $\varepsilon 4$ positive N/Y* & $76 / 25$ & $46 / 34$ & $27 / 47$ & $31 / 71$ \\
\hline -Sex W/M & $49 / 52$ & $26 / 54$ & $28 / 46$ & $43 / 59$ \\
\hline
\end{tabular}

Table 1. Demographics and clinical variables. Means, parentheses $=$ standard deviations. $\mathrm{HC}=$ healthy controls. MCI-S = MCI participants that are clinically stable across 36 months of follow-up. MCI-P = MCI participants that progress to $\mathrm{AD}$ within 36 months of follow-up. $\mathrm{AD}=$ Alzheimer's disease. $\mathrm{MCI}=$ mild cognitive impairment. MMSE = Mini Mental State Examination. AVLT = Auditory Verbal Learning Test. $\mathrm{p}$-tau $=$ phosphorylated tau. $\mathrm{t}$-tau $=$ total tau. $\mathrm{N}=\mathrm{No} / \mathrm{Y}=$ Yes. $\mathrm{W}=$ Women. $\mathrm{M}=$ Men. ${ }^{*} \mathrm{p}<0.01$.

measures (Wechsler Memory Scale-Revised (Logical Memory II subscale; maximum score of 25)) ${ }^{17}$, and activities of daily living (ADL). On the Mini Mental State Examination (MMSE; total score = 30) the range for HC was 24-30, and the education-adjusted cut-off score for Logical Memory II was based on education established as $\geq 9$ for 16 years of education, $\geq 5$ for $8-15$ years, and $\geq 3$ for $0-7$ years. The subjects with MCI had memory complaints, a CDR score of 0.5 , mild impairments on objective cognitive measures (i.e., amnestic deficits), and no significant impairments in ADL. On MMSE the range for MCI was 24-30, and the education-adjusted cut-off score for Logical Memory II was $\leq 8$ for 16 years of education, $\leq 4$ for $8-15$ years, and $\leq 2$ for $0-7$ years. The participants with MCI did not qualify for the diagnosis of AD. Finally, subjects with AD had mild AD with memory complaints, a CDR score $\geq 0.5$, and significant impairments on objective cognitive measures and in ADL. On MMSE the range for $\mathrm{AD}$ was 20-26, and the education-adjusted cut-off score for Logical Memory II was $\leq 8$ for 16 years, $\leq 4$ for $8-15$ years, and $\leq 2$ for $0-7$ years. Individuals with AD met the National Institute of Neurological and Communicative Disorders and Stroke-Alzheimer's Disease and Related Disorders Association criteria for probable $\mathrm{AD}^{18}$. Importantly, all diagnoses were independent of biomarker information. All included participants had available information on CSF biomarkers and MRI atrophy at baseline, as well as longitudinal information regarding diagnostic status. A total of 154 participants with MCI were included in the current study. Of those, 74 progressed to AD (MCI-P) within 36-months, and 80 remained stable in their diagnosis across time (MCI-S). In addition, $101 \mathrm{HC}$ participants and 102 participants with $\mathrm{AD}$ were included for descriptive comparisons. We classified the individuals and compared the groups by assessing the baseline data. For the cognitive characterization, we conducted group comparisons of global cognitive performance as measured with MMSE, and episodic memory as measured with the delayed recall in the Auditory Verbal Learning Test (AVLT; total score $=15$ ). Demographic and clinical variables are presented in Table 1 . The study protocols were approved by the institutional review boards of all included ADNI centres (see supplementary information), and written informed consent (including extensive description of the ADNI) was acquired from all included participants according to the Declaration of Helsinki. All study methods and protocols were performed in accordance with the relevant guidelines and regulations.

Assessment procedure for cerebrospinal fluid biomarkers. For CSF, the $A \beta_{42}$, phosphorylated tau (p-tau), and total tau (t-tau) protein levels were measured from the ADNI baseline aliquots (See Shaw et al., 2009 for detailed information $)^{19}$. In summary, calibration curves were produced for each CSF marker using aqueous buffered solutions that contained the combination of the three CSF markers at concentrations ranging from 56 to $1,948 \mathrm{pg} / \mathrm{ml}$ for recombinant tau, 27 to $1,574 \mathrm{pg} / \mathrm{ml}$ for synthetic $\mathrm{A} \beta_{42}$ peptide, and 8 to $230 \mathrm{pg} / \mathrm{ml}$ for a tau synthetic peptide phosphorylated at the threonine 181 position.

Assessment of the $A / T / N$ classification system. The $A / T / N$ is a three-tailed binary $+/-$ categorization system based on the underlying pathophysiology in each category ${ }^{6}$. We classified the A $\beta$ biomarker "A" with CSF $\mathrm{A} \beta_{42}$, the tau pathology biomarker " $\mathrm{T}$ " with CSF p-tau, and finally the biomarker of neurodegeneration " $N$ " with CSF t-tau. Each individual was rated as either positive (+; i.e., abnormal) or negative (-; i.e., normal) on each biomarker.

The cut-offs of the selected CSF biomarkers have previously been published ${ }^{19}$. In summary, the cut-off values were derived from the comparison between $\mathrm{ADNI} A \mathrm{AD}$ cases and healthy controls. The individual CSF values were considered pathological ( + ) if $\leq 192 \mathrm{pg} / \mathrm{ml}$ for $\mathrm{A} \beta_{42}, \geq 93 \mathrm{pg} / \mathrm{ml}$ for t-tau, and $\geq 23 \mathrm{pg} / \mathrm{ml}$ for $\mathrm{p}$-tau.

Structural MRI and visual rating scales. For MRI, a T1-weighted magnetization-prepared rapid gradient-echo (MPRAGE) sequence was acquired on $1.5 \mathrm{~T}$ scanners with a voxel size of $1.1 \times 1.1 \times 1.2 \mathrm{~mm}^{3}$. All T1-weighted images were visually rated for regional brain atrophy by an experienced radiologist (See Ferreira et al., 2015 for detailed information ${ }^{20}$. In summary, the medial temporal atrophy (MTA) scale scored the degree of atrophy from zero to four ${ }^{21}$. The posterior atrophy (PA) scale scored from zero to three and evaluated atrophy in the posterior cortex (posterior cingulate sulcus, precuneus, parieto-occipital sulcus, and parietal cortex) ${ }^{22}$. Finally, 


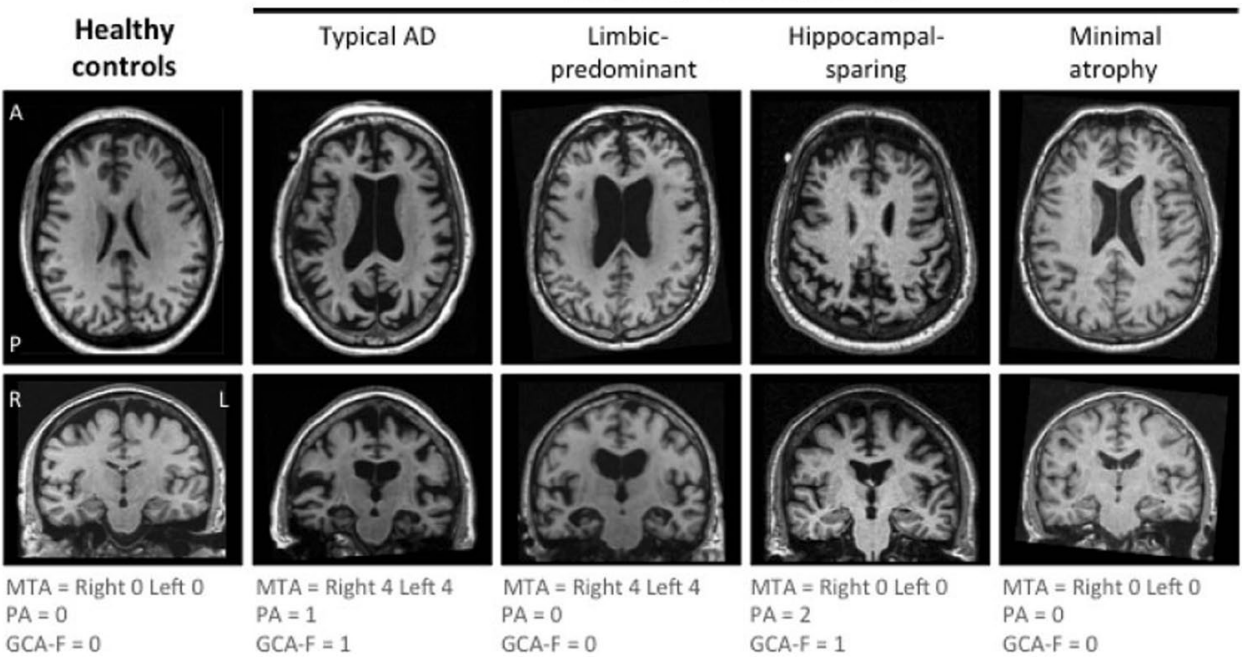

Figure 1. Subtypes of brain atrophy patterns in MCI from visual rating scales Atrophy was measured with the medial temporal atrophy (MTA) scale, the global cortical atrophy-frontal (GCA-F) sub-scale, and the posterior atrophy (PA) scale. The visual rating scales were based on MRI. A score of zero indicates no atrophy. A score from one to four (MTA), and from one to three (GCA-F, and PA) indicates an increasing degree of atrophy. The minimal atrophy group was defined as normal scores on all visual rating scales. The limbic-predominant group was defined as abnormal MTA and normal GCA-F and PA. The typical AD group was defined as abnormal MTA in conjunction with either an abnormal PA or GCA-F, or an abnormal MTA in conjunction with both an abnormal PA and GCA-F. The hippocampal-sparing group was defined as abnormal GCA-F and/or abnormal $\mathrm{PA}$, but normal MTA. $\mathrm{AD}=$ Alzheimer's disease. $\mathrm{L}=$ left. $\mathrm{R}=$ right. $\mathrm{A}=$ anterior. $\mathrm{P}=$ posterior.

the global cortical atrophy scale - frontal subscale (GCA-F) scored from zero to three and evaluated atrophy in the frontal cortex (bordered by the central sulcus, the frontal bone, and the fissure of Sylvius) ${ }^{23}$. Higher scores in the three scales denote end-stage degree of atrophy. Intra-rater reliability (weighted kappa) for MTA was 0.94 and 0.89 in the left and right hemispheres respectively. Equivalent numbers for PA was 0.88, and for GCA-F 0.83.

Classification of brain atrophy patterns. Cut-offs for the visual rating scales have previously been published ${ }^{20}$. In summary, normal versus abnormal cut-off points were determined for each individual in the three visual rating scales. A MTA score $\geq 1,5$ were considered to be abnormal in the age-group $65-74, \geq 2$ for the age-group 75-84, and $\geq 2,5$ for the age-group 85-94. For PA and GCA-F, a score $\geq 1$ was always considered abnormal irrespective of age (age-correction did not improve diagnostic accuracy in cut-offs derivation).

The four atrophy patterns were created using the combination of MTA, PA, and GCA- $\mathrm{F}^{14,24}$, and have previously been described in relation to $\mathrm{AD}^{13}$. The atrophy patterns for each study group are illustrated in Fig. 1. In summary, the minimal-atrophy pattern was defined as no evidence of visual brain atrophy according to the above-mentioned rating scales cut-offs. The limbic-predominant pattern was defined as an abnormal MTA, and a normal PA and GCA-F. The typical AD pattern was defined as an abnormal MTA in conjunction with either an abnormal PA or GCA-F, or an abnormal MTA in conjunction with both an abnormal PA and GCA-F. Finally, the hippocampal-sparing pattern was defined as a normal MTA and either an abnormal PA or GCA-F, or both an abnormal PA and GCA-F.

Statistical analyses. For univariate comparisons of quantitative variables, independent one-way ANOVA/ ANCOVAs were conducted with additional post-hoc follow-ups. The post-hoc analyses were Hochberg corrected for number of comparisons. Chi-square tests were conducted for categorical variables. A p-value $<0.05$ was deemed statistically significant. Descriptive data are presented as mean (standard deviation), and percentages. Prevalence and incidence rate for AD with a 95\% confidence interval (CI) were calculated. Cox regression analyses were conducted to examine the association between $\mathrm{A} / \mathrm{T} / \mathrm{N}$ and brain atrophy characteristics in MCI at baseline and the development of AD. The Hazard ratios (HR) are presented with $95 \%$ CI and p-values. Statistical analyses were performed with the use of IBM SPSS statistics 23.

\section{Results}

The baseline characteristics for HC, MCI-S, MCI-P, and AD are displayed in Table 1. There were no significant group differences regarding age $\left(\mathrm{F}_{3,353}=0.39, \mathrm{p}=0.76\right)$ or years of education $\left(\mathrm{F}_{3,353}=1.68, \mathrm{p}=0.17\right)$. The neuropsychological measures revealed group differences for global cognitive performances measured with MMSE $\left(\mathrm{F}_{3,353}=19.62, \mathrm{p}<0.01\right)$ and episodic memory measured with AVLT delayed recall $\left(\mathrm{F}_{3,353}=99.01\right.$, $\mathrm{p}<0.01)$. Post-hoc analyses showed that all groups differed significantly between each other regarding MMSE and AVLT, except for MCI-P and AD that had similar performances in AVLT $(\mathrm{p}=0.86)$. There were also group differences regarding the CSF biomarkers $A \beta_{42}\left(F_{3,353}=36.99, p<0.01\right)$, p-tau $\left(F_{3,353}=22.09, p<0.01\right)$, and t-tau $\left(F_{3,353}=26.01, p<0.01\right)$. Post-hoc analyses showed that $A \beta_{42}$ levels significantly differed between groups except 


\section{Healthy controls}

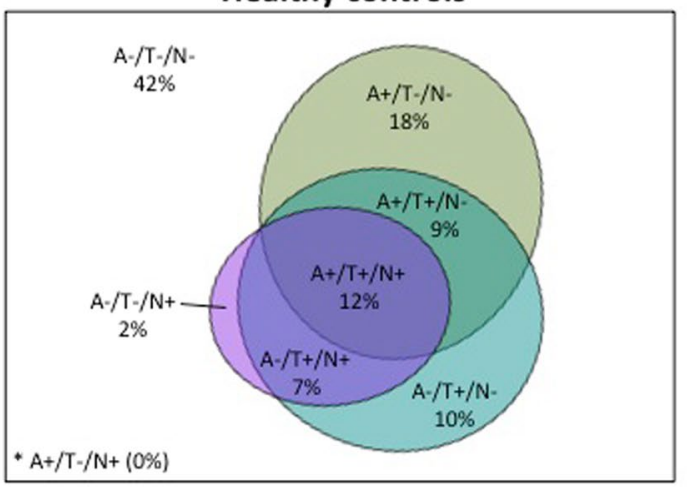

$\mathrm{MCl}-\mathrm{P}$

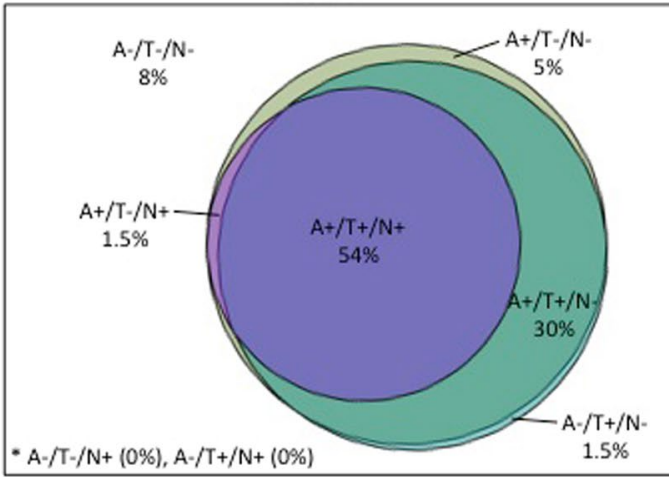

MCl-S

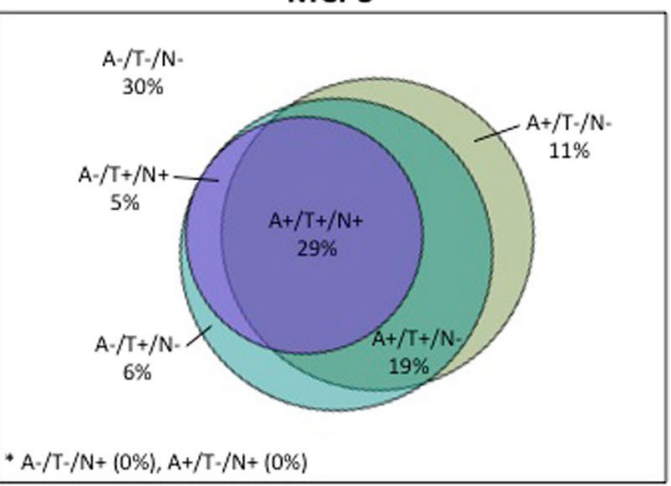

$A D$

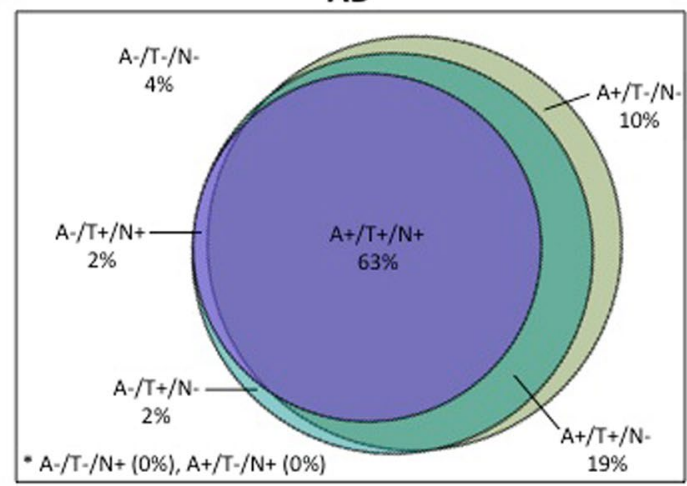

Figure 2. Prevalence of each A/T/N group Percentages of participants in each ATN group for healthy controls, MCI-S, MCI-P, and AD. HC = healthy controls. MCI-S = MCI participants that are clinically stable across time. $\mathrm{MCI}-\mathrm{P}=\mathrm{MCI}$ participants that progress to $\mathrm{AD} . \mathrm{AD}=$ Alzheimer's disease. $\mathrm{MCI}=$ mild cognitive impairment. $\mathrm{CSF}=$ cerebrospinal fluid. $\mathrm{A}-=\mathrm{CSF} \mathrm{A} \beta$ normal. $\mathrm{A}+=\mathrm{CSF} \mathrm{A} \beta$ abnormal. $\mathrm{T}-=\mathrm{CSF}$-tau normal. $\mathrm{T}+=\mathrm{CSF}$ p-tau abnormal. $\mathrm{N}-=$ CSF t-tau normal. $\mathrm{N}+=\mathrm{t}$-tau abnormal.

for MCI-P and AD that had similar values $(\mathrm{p}=0.98)$. For $\mathrm{p}$-tau, post-hoc analyses showed significant group differences except for HC and MCI-S that had similar values $(\mathrm{p}=0.13)$, as well as MCI-P and AD $(\mathrm{p}=0.99)$. For t-tau, post-hoc analyses showed significant group differences except for HC and MCI-S that had similar values $(\mathrm{p}=0.10)$, as well as MCI-P and AD $(\mathrm{p}=0.96)$. The sex distribution was rather similar among groups $\left(\mathrm{X}^{3}=5.11\right.$, $\mathrm{p}=0.16)$. Individuals with the APOE $\varepsilon 4$ genotype were more common in MCI-P and AD than in MCI-S and HC $\left(\mathrm{X}^{3}=48.62, \mathrm{p}<0.01\right)$. The longitudinal examinations showed that 74 of the 154 participants $(48 \%)$ with MCI progressed to AD (MCI-P) whereas 80 participants remained stable across time (MCI-S). This percentage corresponds to an incidence rate of 160 per 1000 individuals with MCI that progress yearly to AD (95\% CI, 127-194).

A/T/N assessment. The descriptive results of the A/T/N classification are displayed in Fig. 2 (see also supplementary material for specific baseline information on the comparison between $\mathrm{A}-/ \mathrm{T}-/ \mathrm{N}-$ or $\mathrm{A}+/ \mathrm{T}+/$ $\mathrm{N}+\mathrm{MCI}$ subjects). Overall, the overlap between amyloid pathology, tangle pathology, and neurodegeneration increased with disease progression, being extensive in MCI-P and AD, and inferior in MCI-S and HC. In particular, participants with a positive "A" biomarker were more highly represented in AD (92\%) and MCI-P (91\%) than in MCI-S (59\%) and HC (39\%). Of the 114 MCI-participants with positive "A", 67 progressed to AD (59\%), which correspond to an incidence rate of 196 per 1000 individuals with MCI that progress yearly to AD (95\% CI, 154-238). Furthermore, an overall positive $A / T / N$ classification (i.e., $A+/ T+/ N+$ ) was more common in $A D$ (63\%) and MCI-P (54\%) than in MCI-S (28\%) and HC (12\%). In contrast, an overall negative A/T/N classification (i.e., $\mathrm{A}-/ \mathrm{T}-/ \mathrm{N}-$ ) was more common in HC (43\%) and MCI-S (31\%) than in MCI-P (8\%) and AD (4\%). As illustrated in Fig. 3, being classified as MCI at baseline with an $A+/ T+/ N+(n=63)$ score increased the risk $(\mathrm{HR}=3.54)$ of progressing to $\mathrm{AD}$ compared with an $\mathrm{A}-/ \mathrm{T}-/ \mathrm{N}-(\mathrm{n}=30)$ score $(95 \% \mathrm{CI}=1.50-8.37$; $\mathrm{p}<0.01)$. In addition, being classified with a positive " $\mathrm{A}$ " $(\mathrm{n}=114)$ biomarker (irrespective of T and N classification) increased the risk ( $\mathrm{HR}=3.84)$ of progressing to AD compared with a negative " $\mathrm{A}$ " $(\mathrm{n}=40)$ biomarker $(95 \% \mathrm{CI}=1.76-8.37 ; \mathrm{p}<0.01)$. As a control analysis, we added the baseline scores for AVLT delayed recall as a covariate to the model. The result showed that the HR for the "A" biomarker was still high (3.07) and significant $(\mathrm{p}<0.01)$.

Characteristics of atrophy patterns in $\mathrm{MCl}$. The demographics and clinical variables for different atrophy patterns in MCI are displayed in Table 2 . There was a significant group difference regarding age $\left(\mathrm{F}_{7,146}=3.66\right.$, $\mathrm{p}<0.01)$ but not education $\left(\mathrm{F}_{7,146}=1.73, \mathrm{p}=0.11\right)$. Post-hoc analyses showed that MCI-S with minimal-atrophy 
A.

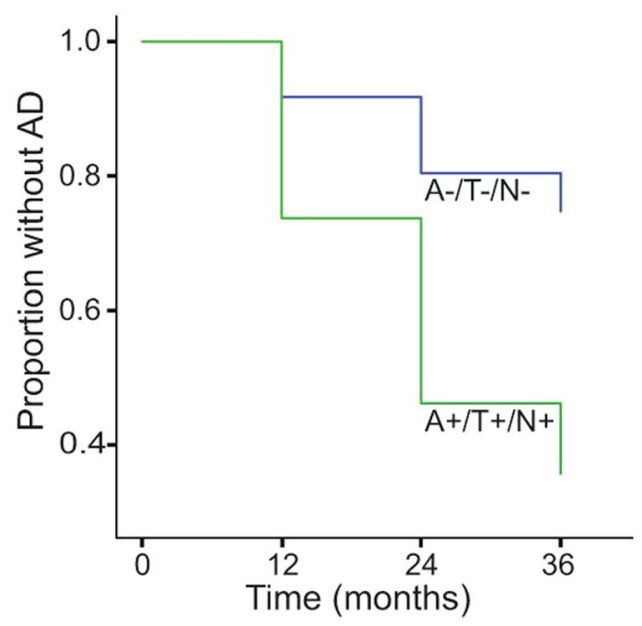

B.

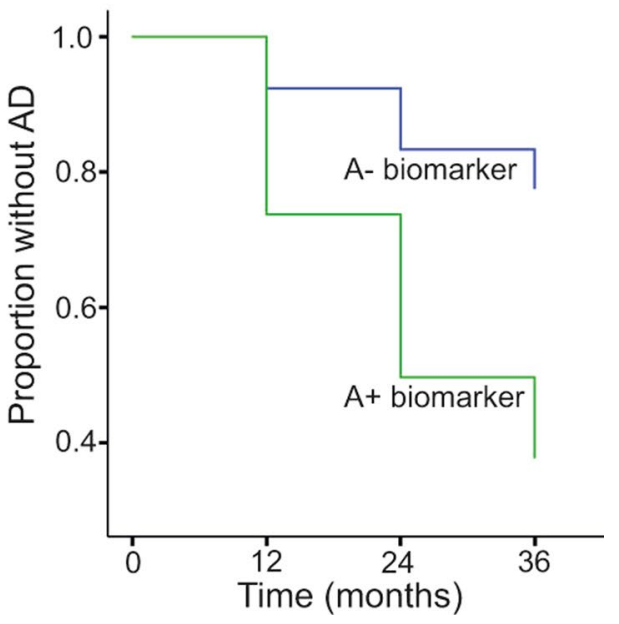

Figure 3. Survival curves of different $\mathrm{A} / \mathrm{T} / \mathrm{N}$ groups in MCI Showing survival curves that illustrate time of progression to $\mathrm{AD}$ for participants with $\mathrm{MCI}$ at baseline with either $(\mathrm{A})$ an $\mathrm{A}-/ \mathrm{T}-/ \mathrm{N}-$ (blue; $\mathrm{n}=30$; mean duration $=28.8$ months, $\mathrm{SD}=9.8)$ or an $\mathrm{A}+/ \mathrm{T}+/ \mathrm{N}+($ green; $\mathrm{n}=63$; mean duration $=25.5$ months, $\mathrm{SD}=9.8)$ pattern, or $(B)$ a negative (blue; $\mathrm{n}=40$; mean duration $=28.8$ months, $\mathrm{SD}=10.1$ ) or a positive (green; $\mathrm{n}=114$; mean duration $=25.0, \mathrm{SD}=9.9$ ) "A" biomarker (irrespective of " $\mathrm{T}$ " and "N" classification).

were younger than both MCI-S with Limbic-predominant atrophy and MCI-P with typical AD. The neuropsychological testing revealed group differences for both global cognitive performances measured with MMSE $\left(\mathrm{F}_{7,146}=2.71, \mathrm{p}=0.01\right)$ and episodic memory measured with AVLT delayed recall $\left(\mathrm{F}_{7,146}=3.33, \mathrm{p}<0.01\right)$. The significant effects on MMSE and AVLT remained after including age and education as covariates. Post-hoc analyses showed no significant group differences on MMSE, but MCI-S with minimal-atrophy performed significantly better on AVLT than MCI-P with limbic-predominant atrophy. There were also group differences regarding the CSF biomarkers $A \beta_{42}\left(F_{7,146}=3.76, p<0.01\right)$, p-tau $\left(F_{7,146}=3.04, p<0.01\right)$, and t-tau $\left(F_{7,146}=3.21, p<0.01\right)$. Post-hoc analyses showed that $A \beta_{42}$ levels were significantly higher in MCI-S with hippocampal-sparing pattern than both MCI-P with minimal-atrophy and MCI-P with a typical AD pattern. For p-tau, the levels were significantly lower for MCI-S with limbic-predominant pattern than both MCI-P with minimal-atrophy, and MCI-P with the hippocampal-sparing pattern. For t-tau, the levels were significantly lower for MCI-S with limbic-predominant atrophy than MCI-P with minimal-atrophy. Of all atrophy groups, MCI-P with minimal-atrophy had the lowest levels of $\mathrm{A} \beta_{42}$, as well as the highest levels of $\mathrm{p}$-tau and $\mathrm{t}$-tau. The distribution regarding gender $\left(\mathrm{X}^{7}=4.16\right.$, $\mathrm{p}=0.76)$ and $\mathrm{APOE} \varepsilon 4\left(\mathrm{X}^{7}=7.47, \mathrm{p}=0.38\right)$ were rather similar between groups.

$\mathrm{A} / \mathrm{T} / \mathrm{N}$ classification on atrophy patterns in $\mathrm{MCl}$. The $\mathrm{A} / \mathrm{T} / \mathrm{N}$ distribution of different atrophy patterns in $\mathrm{MCI}$ is illustrated in Fig. 4. Overall, amyloid positive $(\mathrm{A}+)$ individuals showed a staging pattern where the $\mathrm{A}+/$ $\mathrm{T}+/ \mathrm{N}+$ pattern was most common, followed by the $\mathrm{A}+/ \mathrm{T}+/ \mathrm{N}-$ pattern, and finally the $\mathrm{A}+/ \mathrm{T}-/ \mathrm{N}-$ pattern was less common. Only one individual had an $A+/ T-/ N+$ pattern. In MCI-S, $28 \%(n=23)$ of those that had the minimal-atrophy or hippocampal-sparing pattern also had a negative "A" biomarker. The equivalent number for MCI-P was $3 \%(n=2)$, and the proportion comparison between MCI-S and MCI-P was significant $\left(\mathrm{X}^{1}=25.00\right.$, $\mathrm{p}<0.01)$. In addition, no individual in the MCI-P group with minimal-atrophy had a negative " $\mathrm{A}$ " biomarker, whereas the equivalent number in MCI-S was $15 \%(n=12)$. An overall positive $A / T / N$ classification (i.e., $A+l$ $\mathrm{T}+/ \mathrm{N}+$ ) was common in all MCI-P atrophy patterns. Notably, the $\mathrm{A}+/ \mathrm{T}+/ \mathrm{N}+$ pattern in MCI-P was more frequent in the minimal-atrophy pattern $(\mathrm{n}=14)$ than the Limbic-predominant $(\mathrm{n}=10)$ and the Typical AD pattern $(\mathrm{n}=11)$ subgroups.

As illustrated in Fig. 5a, a survival curve comparison between the different atrophy patterns at baseline, showed that the group with typical AD pattern $(n=29)$ had a HR of 2.99 times $(p<0.01)$ that of the group with hippocampal-sparing pattern $(n=27$; i.e., reference group due to slowest disease progression $)$. In addition, the group with limbic-predominant pattern $(n=46)$ had a HR of 1.98 times $(p=0.079)$ that of the group with hippocampal-sparing pattern. When adding the "A" biomarker information (i.e., if the participant had an A- or an A+ classification) and age (that was significant in the ANOVA) to the model, the " $\mathrm{A}$ " biomarker covariate had a HR of $3.40(\mathrm{p}<0.01)$ indicating that exposure to the covariate increased the risk of progressing to AD. The age covariate had a HR of $1.00(\mathrm{p}=0.87)$ indicating no effect on the outcome. In addition, when only including individuals with A+ biomarkers in the model, a survival curve comparison (Fig. 5b) between different atrophy patterns at baseline, showed that the group with typical AD pattern had a HR of 1.96 times that of the group with hippocampal-sparing pattern, but it did not render in statistical significance $(\mathrm{p}=0.14)$.

\section{Discussion}

We recognize that the $\mathrm{A} / \mathrm{T} / \mathrm{N}$ biomarker system is a useful classification scheme in the MCI population. Importantly, the addition of different brain atrophy patterns accumulated the diagnostic substrate and increased 


\begin{tabular}{|c|c|c|c|c|c|c|c|c|}
\hline & $\begin{array}{l}\text { MCI-SM- } \\
\text { A }(\mathbf{n}=31)\end{array}$ & $\begin{array}{l}\text { MCI-S } \\
\text { L-P }(n=21)\end{array}$ & $\begin{array}{l}\text { MCI-ST-AD } \\
(\mathbf{n}=10)\end{array}$ & $\begin{array}{l}\begin{array}{l}\text { MCI-S H-S } \\
(n=18)\end{array} \\
\end{array}$ & $\begin{array}{l}\text { MCI-P M-A } \\
(\mathrm{n}=21)\end{array}$ & $\begin{array}{l}\text { MCI-P } \\
\text { L-P }(n=25)\end{array}$ & \begin{tabular}{|l|} 
MCI-P \\
T-AD $(n=19)$
\end{tabular} & $\begin{array}{l}\text { MCI-P } \\
\text { H-S }(n=9)\end{array}$ \\
\hline Age** & $70,81(7,05)$ & $76,94(5,15)$ & $77,16(8,37)$ & $76,49(6,38)$ & $71,17(7,89)$ & $73,97(6,30)$ & $77,26(5,66)$ & $77,95(8,76)$ \\
\hline Education & $15,84(2,48)$ & $17,00(2,61)$ & $17,70(2,26)$ & $14,83(3,60)$ & $15,05(3,37)$ & $16,24(3,01)$ & $15,95(1,99)$ & $14,89(4,57)$ \\
\hline MMSE* & $27,58(1,52)$ & $26,71(1,45)$ & $25,90(1,66)$ & $27,78(1,70)$ & $26,67(2,01)$ & $26,36(1,58)$ & $26,68(2,11)$ & $26,00(1,23)$ \\
\hline AVLT del** & $3,87(3,45)$ & $2,81(3,12)$ & $1,90(2,13)$ & $3,78(3,61)$ & $1,48(1,97)$ & $1,36(2,20)$ & $1,47(2,20)$ & $1,33(1,73)$ \\
\hline $\mathrm{A} \beta_{42} * *$ & $172,43(56,12)$ & $168,75(57,49)$ & $162,29(50,82)$ & $206,73(60,01)$ & $137,45(22,52)$ & $150,03(32,12)$ & $145,22(48,80)$ & $168,92(51,51)$ \\
\hline Abnormal A $\beta_{42}$ & $61 \%$ & $67 \%$ & $70 \%$ & $39 \%$ & $100 \%$ & $92 \%$ & $84 \%$ & $78 \%$ \\
\hline p-tau** & $33,19(17,90)$ & $27,62(15,30)$ & $32,30(15,38)$ & $28,56(12,36)$ & $45,05(14,82)$ & $34,72(12,93)$ & $42,79(22,02)$ & $39,89(15,23)$ \\
\hline Abnormal p-tau & $65 \%$ & $52 \%$ & $70 \%$ & $50 \%$ & $95 \%$ & $84 \%$ & $79 \%$ & $78 \%$ \\
\hline $\mathrm{t}$-tau** & $88,16(43,58)$ & $79,65(47,87)$ & $99,80(43,67)$ & $81,82(37,79)$ & $129,77(54,98)$ & $93,93(30,19)$ & $124,36(76,22)$ & $125,64(61,91)$ \\
\hline Abnormal t-tau & $39 \%$ & $24 \%$ & $36 \%$ & $40 \%$ & $67 \%$ & $44 \%$ & $58 \%$ & $67 \%$ \\
\hline $\begin{array}{l}\text { APOE } \varepsilon 4 \text { Positive } \\
\text { N/Y }\end{array}$ & $17 / 14$ & $12 / 9$ & $6 / 4$ & $11 / 7$ & $8 / 13$ & $8 / 17$ & $7 / 12$ & $4 / 5$ \\
\hline Gender W/M & $13 / 18$ & $4 / 17$ & $3 / 7$ & $6 / 12$ & $9 / 12$ & $9 / 16$ & $6 / 13$ & $4 / 5$ \\
\hline
\end{tabular}

Table 2. Demographics and clinical variables for the atrophy patterns in MCI. Means, parentheses=standard deviations. MCI-S = MCI participants that are clinically stable across time. MCI-P $=$ MCI participants that progress to $\mathrm{AD}$ at follow-up. MMSE $=$ Mini Mental State Examination. AVLT = Auditory Verbal Learning Test. $\mathrm{N}=\mathrm{No} / \mathrm{Y}=$ Yes. $\mathrm{W}=$ Women $/ \mathrm{M}=$ Men. $\mathrm{M}-\mathrm{A}=$ Minimal atrophy. $\mathrm{L}-\mathrm{P}=$ Limbic Predominant. $\mathrm{T}-\mathrm{AD}=$ Typical Alzheimer's disease. $\mathrm{H}-\mathrm{S}=$ Hippocampal-sparing. $\mathrm{p}$-tau $=$ phosphorylated tau. $\mathrm{t}$-tau $=$ total tau. $* \mathrm{p}<0.05 . * * \mathrm{p}<0.01$.

knowledge of the clinical trajectories. This combined approach could be important for increasing certainty in the diagnostic and prognostic procedures in $\mathrm{MCI}$, and therefore also supportive in targeting good candidates for interventions. We will first discuss the $\mathrm{A} / \mathrm{T} / \mathrm{N}$ classification scheme assessed in individuals with $\mathrm{MCI}$ and then discuss the impact of brain atrophy patterns when added to the $\mathrm{A} / \mathrm{T} / \mathrm{N}$ classification, with a special focus on variability in the "N" domain.

The A/T/N classification. We showed a progression rate from amnestic MCI to AD (47\%) which is consistent $(48 \%)$ with a previous study with the same follow-up time of 36 -months ${ }^{25}$. In addition, we showed that $58 \%$ of the MCI-participants with a positive "A" biomarker progressed to AD which is comparable with a previous study showing that $57 \%$ of an MCI-population with abnormal $\mathrm{A} \beta_{42}$ progressed to $\mathrm{AD}$ during a mean follow-up of 2.2 years $^{26}$. Notably, being classified as MCI with a positive "A" biomarker increased the risk of progressing to AD during a 36-month period in comparison with being classified with a negative "A" biomarker. The effect was evident even after controling for cognitive baseline status. This resembles models of AD-related neurodegeneration, in which amyloid deposition acts as the trigger of further disease progres$\operatorname{sion}^{27}$. In addition, the $\mathrm{A} / \mathrm{T} / \mathrm{N}$ classification showed a stepwise increase in numbers of $\mathrm{A}+/ \mathrm{T}+/ \mathrm{N}+$ profiles from HC (12\%), via MCI-S (29\%), to MCI-P (54\%), and finally the highest number for AD (63\%). Thus, the more advanced stage of the disease, the higher was the prevalence of positive A/T/N biomarkers, as it was a comorbidity of pathophysiological processes. In addition, the MCI subjects with an overall positive A/T/N pattern had a worse episodic memory performance compared with those with an overall negative A/T/N pattern which might reflect a later stage of $\mathrm{MCI}$ for $\mathrm{A}+/ \mathrm{T}+/ \mathrm{N}+$. Interestingly, we showed that MCI-P had similar $\mathrm{A} / \mathrm{T} / \mathrm{N}$ classification patterns to the group with $\mathrm{AD}$. A positive "A" biomarker was represented in more than ninety percent in both MCI-P and AD. However, a positive "A" biomarker was also present in MCI-S (59\%) and HC (39\%), but to a lesser degree. Thus, there was a considerable amount of individuals in HC and MCI-S with evidence of $\mathrm{AD}$-like pathology. However, $\mathrm{A} \beta_{42}$ deposition increases with age and is also apparent in healthy individuals ${ }^{28,29}$. Recently, an $\mathrm{A} / \mathrm{T} / \mathrm{N}$ classification in cognitively normal individuals showed that the $\mathrm{A}+/ \mathrm{T}+/$ $\mathrm{N}+$ prevalence increased continuously with age ${ }^{30}$. Notably, considering all groups, only one individual had the $\mathrm{A}+/ \mathrm{T}-/ \mathrm{N}+$ profile. This might reflect an individual in an early stage of preclinical $\mathrm{AD}(\mathrm{A}+/ \mathrm{T}-)$ in conjunction with non-AD pathology $(\mathrm{N}+)^{6}$. Thus, in our $\mathrm{A} / \mathrm{T} / \mathrm{N}$ classification of MCI, the CSF biomarker patterns seems to generally follow the order of $\mathrm{A}+$, then $\mathrm{T}+$, and finally $\mathrm{N}+$. As in healthy aging individuals $\mathrm{s}^{30}$, the biomarker sequence of $\mathrm{A}+/ \mathrm{T}-/ \mathrm{N}-$ to $\mathrm{A}+/ \mathrm{T}+/ \mathrm{N}-$ to $\mathrm{A}+/ \mathrm{T}+/ \mathrm{N}+$ is also noted in the current study of $\mathrm{MCI}$ and might represents the pre-dementia AD staging pattern ${ }^{7}$. However, ADNI is a highly selective cohort and the $\mathrm{A}+/ \mathrm{T}-/ \mathrm{N}+$ profile might be more commonly occurring in clinical settings with more diverse pathology and clinical trajectories. Importantly, longer follow-ups are needed to increase knowledge on the staging evolvement in healthy individuals as well as in MCI to confirm the $\mathrm{A} / \mathrm{T} / \mathrm{N}$ progression.

Furthermore, we showed that MCI with a negative "A" biomarker was more common in MCI-S than in MCI-P. Despite that, we reported that seven individuals with MCI and a negative " $\mathrm{A}$ " biomarker progressed to AD within 36 months. Of those seven, six had an $\mathrm{A}-/ \mathrm{T}-/ \mathrm{N}-$ pattern, and one an $\mathrm{A}-/ \mathrm{T}+/ \mathrm{N}-$ pattern. Interestingly, when we added information on atrophy patterns to those profiles, we demonstrated that five individuals had an abnormal MTA score (two individuals with a limbic-predominant pattern and three with a typical AD pattern), and the additional two had abnormal cortical atrophy (both with a hippocampal-sparing pattern). Thus, the biomarker profiles of these individuals might be related to suspected non-AD pathophysiology (SNAP) ${ }^{6,27}$, that has previously been suggested to target individuals without evidence of amyloid accumulation but with evidence of neuronal injury. Possibly, such a pattern might also resemble neurodegeneration due to non-AD pathology $\mathrm{y}^{30}$. 


\section{$\mathrm{MCl}-\mathrm{S}$}

\section{A. A- classifications}

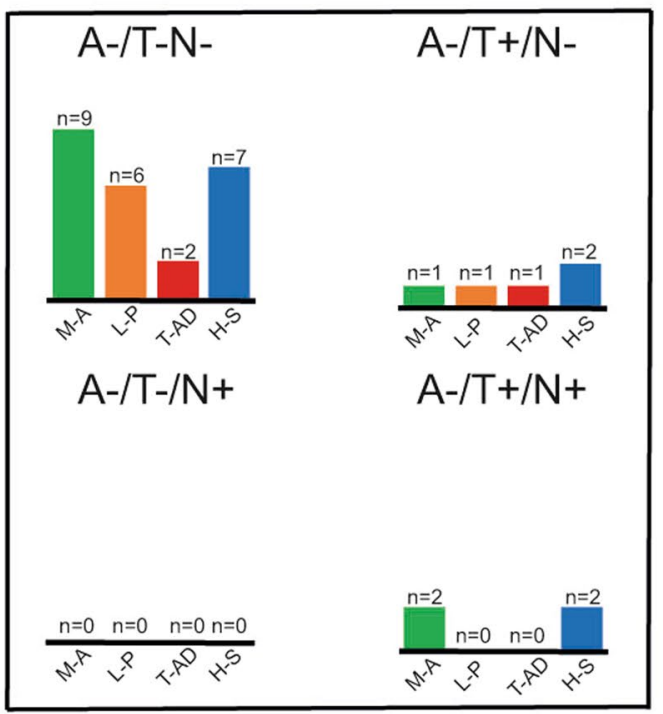

B. A+ classifications

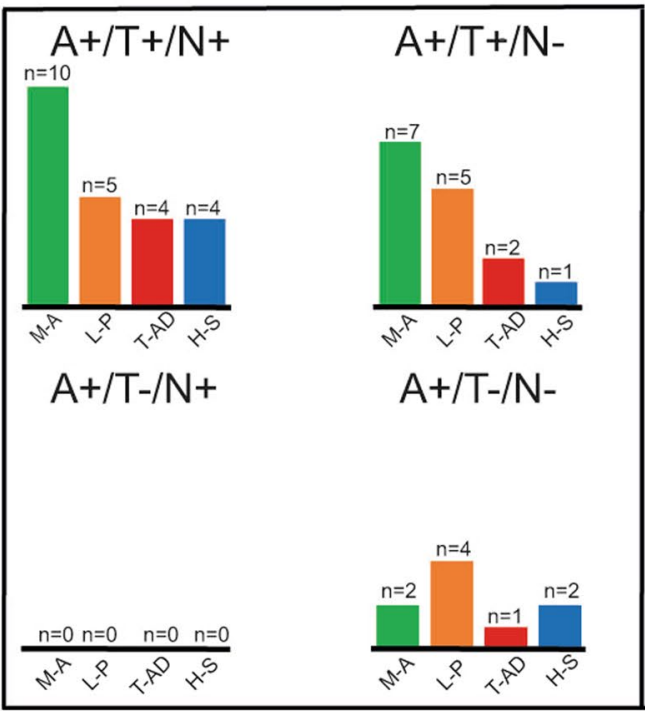

C. A- classifications

\section{$\mathrm{MCl}-\mathrm{P}$}

\begin{tabular}{|c|c|}
\hline A-/T-N- & $\mathrm{A}-/ \mathrm{T}+/ \mathrm{N}-$ \\
\hline$n=0$ & $\mathrm{n}=0 \quad \mathrm{n}=0 \quad{ }^{\mathrm{n}=1} \mathrm{n}=0$ \\
\hline 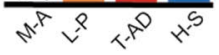 & $4 v^{8} x_{8} x_{2}$ \\
\hline A-/T-/N+ & $\mathrm{A}-/ \mathrm{T}+/ \mathrm{N}+$ \\
\hline$n=0 \quad n=0 \quad n=0 \quad n=0$ & $n=0 \quad n=0 \quad n=0 \quad n=0$ \\
\hline $4_{8} v^{8} x_{0} x_{2}$ & $3 x v_{8} x_{0} x_{2}$ \\
\hline
\end{tabular}

D. A+ classifications

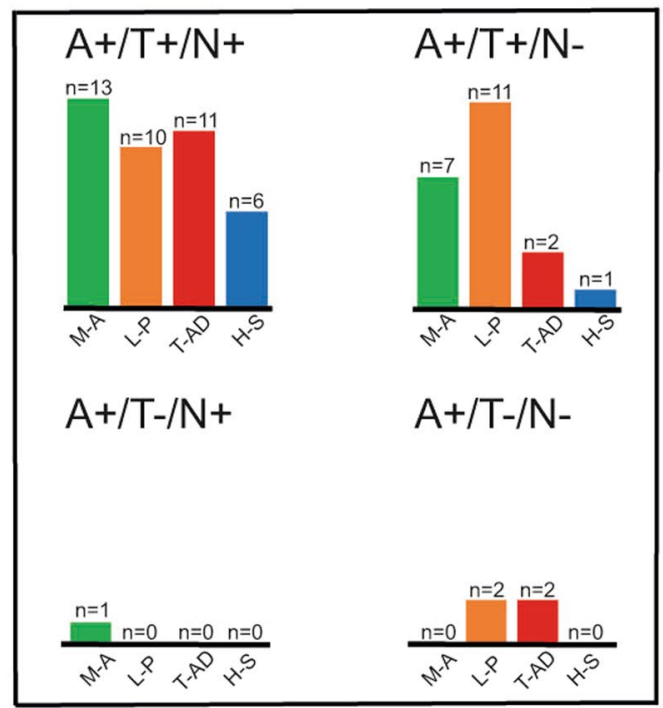

Figure 4. Number of A/T/N classified MCI-participants with different atrophy patterns Number of participants with MCI-S and an A-classifications (A), MCI-S and an A+ classification (B), MCI-P and an $\mathrm{A}-$ classifications $(\mathbf{C})$, and MCI-P and an A+classification (D) pattern. MCI-S $=$ MCI participants that are clinically stable across time. $\mathrm{MCI}-\mathrm{P}=\mathrm{MCI}$ participants that progress to $\mathrm{AD}$. $\mathrm{A}=\mathrm{A} \beta$ biomarker. $\mathrm{T}=$ tau pathology biomarker. $\mathrm{N}=$ the biomarker of neurodegeneration. $\mathrm{M}-\mathrm{A}=$ Minimal atrophy. $\mathrm{L}-\mathrm{P}=\mathrm{Limbic}$ Predominant. $\mathrm{T}-\mathrm{AD}=$ Typical Alzheimer's disease. $\mathrm{H}-\mathrm{S}=$ Hippocampal-sparing.

In addition, no $\mathrm{MCI}$ individual with an $\mathrm{A}-/ \mathrm{T}-/ \mathrm{N}-$ profile and minimal-atrophy progressed to $\mathrm{AD}$ within the studied time-frame. Thus, an MCI diagnosis with an overall negative A/T/N classification in addition to a pattern of minimal-atrophy does not seem to represent $\mathrm{MCI}$ due to $\mathrm{AD}^{31}$ or prodromal $\mathrm{AD}$, and the clinical cognitive symptoms are probable due to other underlying causes ${ }^{8}$. All these findings suggest that in MCI individuals with a negative "A" biomarker, it is crucial to investigate the patterns of atrophy on MRI as well, which may have higher diagnostic utility than the CSF T-Tau levels.

The addition of brain atrophy patterns. Notably, the $\mathrm{A}+/ \mathrm{T}+/ \mathrm{N}+$ pattern was more common in the MCI-P groups with minimal atrophy and hippocampal sparing pattern than the MCI-P groups with limbic predominant 
A.

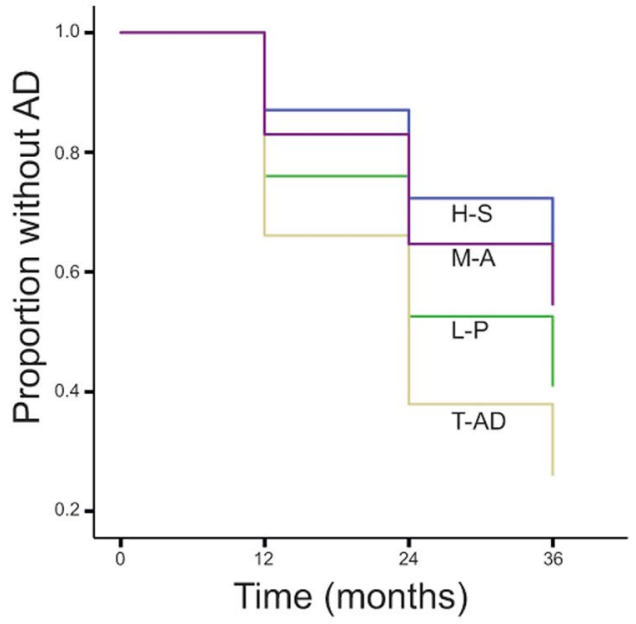

B.

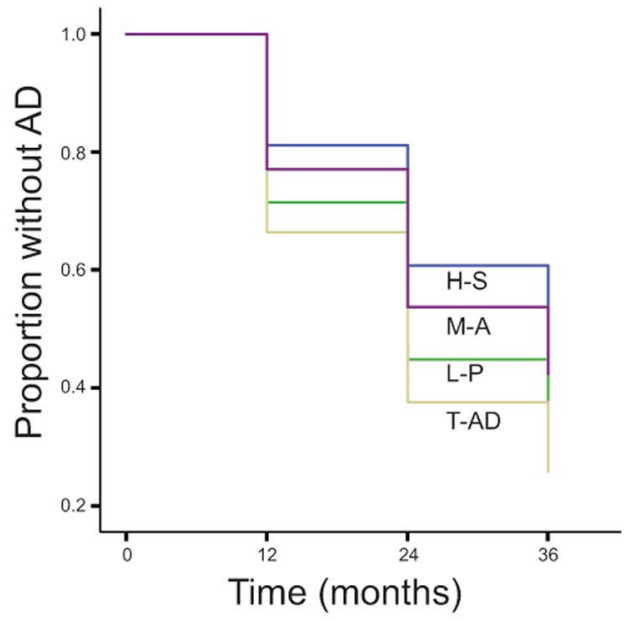

Figure 5. Survival curves of different brain atrophy patterns in MCI (A) Showing survival curves that illustrate time to AD for participants with MCI at baseline with either $\mathrm{M}-\mathrm{A}=$ Minimal atrophy (purple), $\mathrm{L}-\mathrm{P}=\mathrm{Limbic}$ Predominant atrophy (green), T-AD = Typical Alzheimer's disease atrophy (beige), or H-S = Hippocampalsparing pattern (blue). (B) Showing survival curves as in A, but only including individuals with A+ biomarkers in the model.

and typical AD patterns. The minimal atrophy and hippocampal sparing groups had lower levels of education, and the minimal atrophy group was also younger than the other groups, as recently reported in AD dementia ${ }^{32}$. In addition, MCI-P with minimal atrophy had the lowest levels of $\mathrm{A} \beta_{42}$ and the highest levels of $\mathrm{p}$-tau and $\mathrm{t}$-tau. This is in conjunction with what has previously been reported in groups with minimal atrophy in an AD population $^{13}$. Also, the step-wise addition of $\mathrm{p}$-tau (as a neurodegeneration marker) to cognitive measures has previously shown to improve risk stratification compared to hippocampal volumes (derived from MRI) in subjects with $\mathrm{MCI}^{33}$. Individuals with $\mathrm{MCI}$ and a minimal atrophy pattern in conjunction with abnormal CSF $(\mathrm{A}+/ \mathrm{T}+/ \mathrm{N}+)$ could therefore be good candidates for interventions due to their absence of irreversible brain atrophy, together with young age, less cognitive impairment at baseline (i.e., AVLT delayed), and slow cognitive decline across time ${ }^{13}$.

Additionally, as suggested by Jack et al. ${ }^{6}$, we described the "N" category with only one biomarker ( $\mathrm{t}$-tau) in the current study. Another established marker to describe the " $\mathrm{N}$ " category is brain atrophy derived from MRI. When comparing the " $\mathrm{N}$ " classification with the atrophy patterns, several individuals with MCI showed inconsistency regarding the " $\mathrm{N}$ " binary classification and the MRI character of atrophy. For example there were individuals with MCI-P and $\mathrm{A}+/ \mathrm{T}+/ \mathrm{N}+$ in conjunction with minimal atrophy (i.e., $\mathrm{N}-$ according to the visual rating scales). This highlights an incongruity between CSF t-tau and brain atrophy in MRI. Those conflicting results have been emphasised previously, showing that neurodegeneration biomarkers are modestly correlated ${ }^{27}$. In the absence of overt neuronal injury this might be related to increased neuronal secretion of tau in response to amyloid pathology, and that CSF t-tau and p-tau concentrations do not reflect neurodegeneration and tangle pathology directly $^{34}$. These neurons may eventually accumulate tangle pathology and degenerate, but that would become evident in MRI many years later. In addition, this emphasises that individuals with MCI display heterogeneous neurodegeneration patterns as reflected on both $\mathrm{MRI}^{35}$, and $\mathrm{CSF}^{36}$, and that different biomarkers might become abnormal at different stages of the disease progression, or never coincide ${ }^{37}$.

The MCI individuals with a positive "A" biomarker in conjunction with amnestic impairment should be considered as prodromal AD according to the IWG criteria ${ }^{8}$, and as MCI due to AD according to the NIA-AA criteria ${ }^{31}$. In agreement, the addition of atrophy patterns to the "A" biomarker information, at least to some degree, enhanced confidence to the MCI prognoses in the current study. The progression rate from MCI to AD varies and annual rates between $4-36 \%$ have been reported ${ }^{38}$. Thus, we cannot with certainty confirm that MCI-S individuals in the current study will progress to AD. However, the MCI-S individuals with a positive "A" biomarker and AD-like atrophy patterns are likely to progress in respect to previous reports. For example, higher rates of AD-like atrophy has been reported in progressive $\mathrm{MCI}^{39,40}$, and amyloid positive $\mathrm{MCI}$ individuals have been shown to be more likely to progress to $\mathrm{AD}$ than amyloid negative $\mathrm{MCI}$ individuals ${ }^{41}$. However, further longitudinal large-scale clinical studies that examine atrophy patterns in conjunction with $\mathrm{A} / \mathrm{T} / \mathrm{N}$ classifications are needed to increase knowledge on different MCI pathways. The current study shows for the first time that this joint strategy holds high promise.

Limitations and future prospects. Since MCI individuals in ADNI are amnestic at entry, a future prospect is to adapt the $\mathrm{A} / \mathrm{T} / \mathrm{N}$ classification scheme and atrophy patterns to MCI participants with non-amnestic clinical presentations. As shown previously, the nature of memory impairment might differ due to the influence of non-memory cognitive functions on memory performance across atrophy patterns in $\mathrm{AD}^{13}$. In the current study, $\mathrm{MCI}$ and $\mathrm{AD}$ are only represented by amnestic deficits which narrows the spectra of atypical AD-related topographies, also due to the unresolved question whether $\mathrm{AD}$ must be represented solely by amnestic impairment ${ }^{42}$. 
In addition, the $\mathrm{A} / \mathrm{T} / \mathrm{N}$ classification scheme needs to be further validated, especially in community samples with MCI subjects. This would make it possible to draw firmer conclusions on the clinical significance of the scheme and first thereafter be able to be applied on MCI subjects in general. Thus, research studies in large-scale clinical settings are important to increase knowledge on this and other related topics.

This study has some additional limitations. Several individuals have CSF values close to the cut-offs. In clinical settings, the values close to the cut-offs have to be carefully interpreted and handled in relation to additional clinical information, as well as additional biomarker information. We only followed the participants for 36 months after their initial diagnosis, and a longer follow-up might have increased the number of MCI participants progressing to $\mathrm{AD}^{43}$. In addition, the sub-group categorization focusing on atrophy patterns in MCI partly rendered in small sample sizes, and a future prospect would therefore be to maximise sample sizes to increase the possibilities for stronger generalizations to the MCI-population.

\section{Conclusions}

We conclude that the A/T/N classification scheme is easy and adaptable on individuals with $\mathrm{MCI}$, and that visual rating scales can be used to identify $\mathrm{AD}$-related atrophy patterns also in MCI. We presented that no individual with MCI progressed to AD if they had a negative " $\mathrm{A}$ " biomarker in conjunction with minimal atrophy. In contrast, several individuals with MCI progressed to AD if they had a positive "A" biomarker in conjunction with minimal atrophy. In addition, many individuals with MCI showed inconsistency in the neurodegeneration domain ("N"), reflecting an incongruity between CSF t-tau levels and brain atrophy on MRI. Thus, we propose that the A/T/N biomarker system should be applied and interpreted together with information about brain atrophy patterns. This joint strategy shows promising sings for increasing certainty in the diagnostic and prognostic procedures in MCI, and can also be supportive in targeting suitable candidates for interventions. Accordingly, implementation in the clinical routine could be justified.

\section{References}

1. Winblad, B. et al. Mild cognitive impairment-beyond controversies, towards a consensus: report of the International Working Group on Mild Cognitive Impairment. J. Intern. Med. 256, 240-246 (2004).

2. Beach, T. G., Monsell, S. E., Phillips, L. E. \& Kukull, W. Accuracy of the Clinical Diagnosis of Alzheimer Disease at National Institute on Aging Alzheimer Disease Centers, 2005-2010. J. Neuropathol. Exp. Neurol. 71, 266-273 (2012).

3. Gamberger, D., Lavrač, N., Srivatsa, S., Tanzi, R. E. \& Doraiswamy, P. M. Identification of clusters of rapid and slow decliners among subjects at risk for Alzheimer's disease. Sci. Rep 7, 1-12 (2017).

4. Hinrichs, C., Singh, V., Xu, G. \& Johnson, S. C. Predictive markers for AD in a multi-modality framework: An analysis of MCI progression in the ADNI population. Neuroimage 55, 574-589 (2011).

5. Mangialasche, F. et al. Classification and prediction of clinical diagnosis of Alzheimer's disease based on MRI and plasma measures of $\alpha-/ \gamma$-tocotrienols and $\gamma$-tocopherol. J. Intern. Med. 273, 602-621 (2013).

6. Jack, C. R., Hampel, H. J., Universities, S., Cu, M. \& Petersen, R. C. A/T/N: An unbiased descriptive classification scheme for Alzheimer disease biomarkers. Neurology 87, 539-547 (2016).

7. McKhann, G. et al. The diagnosis of dementia due to Alzheimer's disease: Recommendations from the National Institute on AgingAlzheimer's Association workgroups on diagnostic guidelines for Alzheimer's disease. Alzheimers Dement. 7, 263-269 (2011).

8. Dubois, B. et al. Advancing research diagnostic criteria for Alzheimer's disease: the IWG - 2 criteria. Lancet Neurol. 13, 615-629 (2014).

9. Harper, L. et al. MRI visual rating scales in the diagnosis of dementia: evaluation in 184 post-mortem confirmed cases. Brain 139, 1211-1225 (2016).

10. Byun, M. S. et al. Heterogeneity of regional brain atrophy patterns associated with distinct progression rates in Alzheimer's disease. PLoS One 10, 1-16 (2015).

11. Mueller, S. G. et al. The Alzheimer's Disease Neuroimaging Initiative. Neuroimaging Clin. N. Am. 15, 869-877 (2005).

12. Jr, C. R. J. et al. The Alzheimer's Disease Neuroimaging Initiative (ADNI): MRI Methods. J. Magn. Reson. Imaging 27, 685-691 (2008).

13. Ferreira, D. et al. Distinct subtypes of Alzheimer's disease based on patterns of brain atrophy: longitudinal trajectories and clinical applications. Sci. Rep 7, 46263 (2017).

14. Murray, M. E. et al. With distinct clinical characteristics: A retrospective study. Lancet Neurol. 10, 785-796 (2011).

15. Petersen, R. C. et al. Alzheimer's Disease Neuroimaging Initiative (ADNI): Clinical characterization. Neurology 74, 201-209 (2010).

16. Morris, J. C. The clinical dementia rating (CDR): current vision and scoring rules Neurology. Neurology 4, 2412-2414 (1993).

17. Wechsler, D. A. Wechsler Memory Scale-Revised. (Psychological Corporation 1987).

18. McKhann, G., Folstein, M., Katzman, R., Price, D. \& Stadlan, E. Clinical diagnosis of Alzheimer's disease: report of the NINCDSADRDA work group under the auspices of Department of Health and Human Services Task Force on Alzheimer's Disease. Neurology 34, 939-944 (1984).

19. Shaw, L. M. et al. Cerebrospinal Fluid Biomarker Signature in Alzheimer's Disease Neuroimaging Initiative Subjects. Ann. Neurol. 65, 403-413 (2009).

20. Ferreira, D. et al. Practical cut-offs for visual rating scales of medial temporal, frontal and posterior atrophy in Alzheimer's disease and mild cognitive impairment. J. Intern. Med. 278, 277-290 (2015).

21. Scheltens, P. et al. Atrophy of medial temporal lobes on MRI in 'probable' Alzheimer's disease and normal ageing: diagnostic value and neuropsychological correlates. J.Neurol.Neurosurg.Psychiatry 55, 967-972 (1992).

22. Koedam, E. L. G. E. et al. Visual assessment of posterior atrophy development of a MRI rating scale. Eur. Radiol. 21, 2618-2625 (2011).

23. Ferreira, D. et al. Quantitative validation of a visual rating scale for frontal atrophy: associations with clinical status, APOEe4, CSF biomarkers and cognition. Eur. Radiol. 26, 2597-2610 (2016).

24. Whitwell, J. L. et al. Neuroimaging correlates of pathologically-defined atypical Alzheimer's disease. Lancet Neurol. 11, 868-877 (2012).

25. Okello, A. et al. Conversion of amyloid positive and negative mci to ad over 3 years: An c-pib pet study symbol. Neurology 73 , 754-760 (2009).

26. Van Rossum, I. A. et al. Injury markers predict time to dementia in subjects with MCI and amyloid pathology. Neurology 79, 1809-1816 (2012).

27. Alexopoulos, P. et al. Limited agreement between biomarkers of neuronal injury at different stages of Alzheimer's disease. Alzheimer's Dement. 10, 684-689 (2014).

28. Rodrigue, K. M. et al. B-Amyloid burden in healthy aging. Regional distribution and cognitive consequences. Neurology 78, 387-395 (2012).

29. Höglund, K. et al. Preclinical amyloid pathology biomarker positivity: effects on tau pathology and neurodegeneration. Transl. Psychiatry 7, e995 (2017).

30. Jack, C. R. et al. Age-specific and sex-specific prevalence of cerebral $\beta$-amyloidosis, tauopathy, and neurodegeneration in cognitively unimpaired individuals aged 50-95 years: a cross-sectional study. Lancet Neurol. 16, 435-444 (2017). 
31. Sperling, R. A. et al. Toward defining the preclinical stages of Alzheimer's disease: Recommendations from the National Institute on Aging- Alzheimer's Association workgroups on diagnostic guidelines for Alzheimer's disease. Alzheimer's Dement 7, $280-292$ (2011).

32. Persson, K. et al. MRI-assessed atrophy subtypes in Alzheimer's disease and the cognitive reserve hypothesis. PLoS One 12, 1-15 (2017).

33. Lange, C. et al. Prediction of Alzheimer's Dementia in Patients with Amnestic Mild Cognitive Impairment in Clinical Routine: Incremental Value of Biomarkers of Neurodegeneration and Brain Amyloidosis Added Stepwise to Cognitive Status. J. Alzheimer's Dis 61, 373-388 (2017).

34. Maia, L. F. et al. Changes inAmyloid- and Tau in the Cerebrospinal Fluid of Transgenic Mice Overexpressing Amyloid Precursor Protein. Sci. Transl. Med. 5, 194re2 (2013).

35. Dong, A. et al. Heterogeneity of neuroanatomical patterns in prodromal Alzheimer's disease: links to cognition, progression and biomarkers. Brain 140, 735-747 (2017).

36. Nettiksimmons, J., DeCarli, C., Landau, S. \& Beckett, L. Biological heterogeneity in ADNI amnestic MCI. Alzheimers. Dement. 10, 511-521 (2014).

37. Frisoni, G. B. et al. Imaging markers for Alzheimer disease: Which vs how. Neurology 81, 487-500 (2013).

38. Bennett, D. A. et al. Natural history of mild cognitive impairment in older persons. Neurology 59, 198-205 (2002).

39. Falahati, F. et al. Monitoring disease progression in mild cognitive impairment: Associations between atrophy patterns, cognition, APOE and amyloid. NeuroImage Clin. 16, 418-428 (2017).

40. Spulber, G. et al. An MRI-based index to measure the severity of Alzheimer's disease-like structural pattern in subjects with mild cognitive impairment. J. Intern. Med. 273, 396-409 (2013).

41. Jack, C. R. et al. Brain beta-amyloid measures and magnetic resonance imaging atrophy both predict time-to-progression from mild cognitive impairment to Alzheimer's disease. Brain 133, 3336-3348 (2010).

42. Morris, J. C. et al. Harmonized diagnostic criteria for Alzheimer's disease: Recommendations. J. Intern. Med. 275, 204-213 (2014).

43. Wallin, A. et al. The Gothenburg MCI study: Design and distribution of Alzheimer's disease and subcortical vascular disease diagnoses from baseline to 6-year follow-up. J. Cereb. Blood Flow Metab. 36, 114-131 (2016).

\section{Acknowledgements}

This project is financially supported by the Swedish Foundation for Strategic Research (SSF), the Swedish Research Council (VR), the Swedish Research Council for Health, Working Life and Welfare, the Strategic Research Programme in Neuroscience at Karolinska Institutet (StratNeuro), the regional agreement on medical training and clinical research (ALF) between Stockholm County Council and Karolinska Institutet, Hjärnfonden, Alzheimerfonden, Stiftelsen Olle Engkvist Byggmästare, and Åke Wibergs Stiftelse. We also thank Birgitta och Sten Westerberg for additional finacial support. Data collection and sharing for this project was funded by the Alzheimer's Disease Neuroimaging Initiative (ADNI) (National Institutes of Health Grant U01 AG024904) and DOD ADNI (Department of Defense award number W81XWH-12-2-0012). ADNI is funded by the National Institute on Aging, the National Institute of Biomedical Imaging and Bioengineering, and through generous contributions from the following: AbbVie, Alzheimer's Association; Alzheimer's Drug Discovery Foundation; Araclon Biotech; BioClinica, Inc.; Biogen; Bristol-Myers Squibb Company; CereSpir, Inc.; Cogstate; Eisai Inc.; Elan Pharmaceuticals, Inc.; Eli Lilly and Company; EuroImmun; F. Hoffmann-La Roche Ltd and its affiliated company Genentech, Inc.; Fujirebio; GE Healthcare; IXICO Ltd.; Janssen Alzheimer Immunotherapy Research \& Development, LLC.; Johnson \& Johnson Pharmaceutical Research \& Development LLC.; Lumosity; Lundbeck; Merck \& Co., Inc.; Meso Scale Diagnostics, LLC.; NeuroRx Research; Neurotrack Technologies; Novartis Pharmaceuticals Corporation; Pfizer Inc.; Piramal Imaging; Servier; Takeda Pharmaceutical Company; and Transition Therapeutics. The Canadian Institutes of Health Research is providing funds to support ADNI clinical sites in Canada. Private sector contributions are facilitated by the Foundation for the National Institutes of Health (www.fnih.org). The grantee organization is the Northern California Institute for Research and Education, and the study is coordinated by the Alzheimer's Therapeutic Research Institute at the University of Southern California. ADNI data are disseminated by the Laboratory for Neuro Imaging at the University of Southern California. Data used in preparation of this article were obtained from the Alzheimers's Disease Neuroimaging Initiative (ADNI) database (adni.loni.usc.edu). As such, the investigators within the ADNI contributed to the design and implementation of ADNI and/or provided data but did not participate in analysis or writing of this report. A complete listing of ADNI investigators can be found at: http://adni.loni.usc.edu/ wp-content/uploads/how to apply/ADNI Acknowledgement Lists.pdf.

\section{Author Contributions}

Dr. Ekman, Dr. Ferreira, and Dr. Westman designed the study and organised the data. All authors analysed and interpreted the data. Dr. Ekman wrote the original draft of the manuscript. All authors critical reviewed and edited the manuscript.

\section{Additional Information}

Supplementary information accompanies this paper at https://doi.org/10.1038/s41598-018-26151-8.

Competing Interests: The authors declare no competing interests.

Publisher's note: Springer Nature remains neutral with regard to jurisdictional claims in published maps and institutional affiliations.

Open Access This article is licensed under a Creative Commons Attribution 4.0 International License, which permits use, sharing, adaptation, distribution and reproduction in any medium or format, as long as you give appropriate credit to the original author(s) and the source, provide a link to the Creative Commons license, and indicate if changes were made. The images or other third party material in this article are included in the article's Creative Commons license, unless indicated otherwise in a credit line to the material. If material is not included in the article's Creative Commons license and your intended use is not permitted by statutory regulation or exceeds the permitted use, you will need to obtain permission directly from the copyright holder. To view a copy of this license, visit http://creativecommons.org/licenses/by/4.0/.

(c) The Author(s) 2018 